\title{
Outcome comparison between radiochemotherapy and surgery in the management of locally advanced non-metastatic laryngeal cancer a single institution experience
}

\begin{abstract}
The treatment of locally advanced non-metastatic laryngeal squamous cell carcinoma is very controversial. Total laryngectomy associated with lymph node dissection and adjuvant radiotherapy with or without chemotherapy is considered the gold standard treatment. The functional impairment on voice and breathing that result from this approach called for discussion of preservation of this organ. Since the publication of the Veterans' Study in 1991 on laryngeal cancer and the confirmation by subsequent randomized trials of an equivalent survival, treatment strategies for advanced laryngeal carcinoma have shown significant changes in favour of an organ-sparing approach by chemoradiotherapy.
\end{abstract}

Purpose: We aim to assess the outcome of locally advanced non-metastatic laryngeal cancer classified as (T3NxM0 -T4NxM0) by comparing the carcinological results and the survival at one and three years between two cohorts of patients, one treated by surgery and the other by organ preservation protocols. Between the two series, we will analyze the carcinological outcomes, local control, local and lymph node recurrence, distant metastases, overall survival, and recurrence-free survival, lymph Node-free survival, and metastatic evolution.

Results: 106 patients were treated for locally advanced squamous cell laryngeal carcinoma of the ENT department and radiation Oncology department of Mohamed VI University hospital between January 2014 and December 2018; Sixty-three patients in surgery group I and forty-three patients in group II went on organ sparing approach by radiochemotherapy. The two groups were compared according to local tumor control, local recurrence, lymph node recurrence, and distant metastasis. Early deaths and patients who were lost to follow-up were excluded from this analysis. The average age was 61 years in the surgery group versus 60 years in the RCC. The male predominance was marked in both treatment groups, 102 were male $(96.23 \%)$ and only 4 female $(3.77 \%$.). $88.7 \%$ were smokers with an average consumption of 26.4 package-years. Only $15 \%$ of our smoking patients reported a withdrawal period estimated at two months on average. Alcohol-smoking synergy was observed in $19 \%$ of cases. In the surgery group, 47 patients or $83.9 \%$ had local tumor control compared to 12 patients or $41.4 \%$ in the radio-chemotherapy group with a statistically significant difference $p<0.0001$. Local recurrence was observed in 8 patients $(14.5 \%)$ in the surgery group against 6 patients $(46.2 \%)$ in the radio-chemotherapy cohort with a $\mathrm{p}=0.02$. We noted that there was a large number of missing data (30 patients) in the radio-chemotherapy group due to the large number of patients who were lost to follow-up, early deaths, and patients who did not progress well after treatment. There was no statistically significant difference between the two groups in terms of lymph node recurrence and metastatic progression. At 1 year, Overall survival was $87.9 \%$ of patients were alive ( $\mathrm{n}=51$ out of 58 ) in the surgery arm versus $60.6 \%$ $(\mathrm{n}=20$ out of 33$)$ in the radio-chemotherapy arm. At 3 years overall survival was $77.5 \%$ for surgery versus $48.4 \%$ for radiotherapy ( $p=0.005$ ).Lymph node

\section{Introduction}

Laryngeal cancer is a frequent location among cancers of the upper aero-digestive tract. Its incidence is constantly evolving with 177420 new cases per year in the world, which corresponds
Volume I 3 Issue 2 - 202 |

\author{
Nimubona Désiré, Benyouness Leilla, \\ El Lanigri Merriam, Diouf Kady, Bounid \\ Oumaima, Darfaoui Mouna, Issam Lalya, El \\ Omrani Abdelhamid, Khouchani Mouna \\ Radiation Oncology Department, Mohamed VI Hospital \\ University, Morocco
} Department, Mohamed VI University Hospital, Marrakech, Morocco, BP 40000, Marrakech, Tel 00212684751089, Email nimubonadesire78@gmail.com

Received: April 18,2021 | Published: April 28, 2021

free recurrence and metastatic free progression at 1 year was $94.5 \%$ in the surgery group compared with $84.6 \%$ for radio-chemotherapy. Survival at 3 years was $85.4 \%$ versus $53.8 \%$ respectively $(\mathrm{p}=0.05)$.In the chemoradiation therapy group, there were 30 missing data due to a large number of deaths and loss of the follow-up during the first year without any indication of the presence or absence of recurrence, compared to 8 missing data for the radiochemotherapy group.

Conclusion: The optimal treatment for advanced squamous cell carcinoma of the larynx is highly controversial. Total laryngectomy associated with cervical lymph node dissection remains the gold standard of treatment but organ-sparing protocols are as effective as surgical therapy. However, in our study, total laryngectomy plus lymph node dissection showed better survival outcomes in terms of locoregional control and significantly increased overall survival and recurrence-free survival. This makes surgery the treatment of choice in the management of locally advanced non-metastatic laryngeal cancer in our single institutional Moroccan setting.

Possible reasons for these results may be poor patient selection, inadequate follow-up, incomplete treatment, and interrupted treatment sessions but also the long delay in consultation. Patients and professionals should be made aware of the small but significant disadvantage of the non-surgical therapy approach as part of the shared decision-making process when selecting treatment. Both surgery and radio-chemotherapy can be effective if the treatment indications are well directed. These indications depend on several many several parameters and should be considered at the multidisciplinary consultation meetings and adapted on a case-by-case basis.

Keywords: locally advanced laryngeal cancer, surgery, chemoradiation, survival

to $1 \%$ of all cancers. It accounts for $2 \%$ of all cancers recorded in Morocco between 2008 and 2012 in the cancer register of the Greater Casablanca region. It is cancer with a high male dominance and the major risk factor is cigarette smoking with an increased risk in case of alcohol/tobacco synergy. Squamous cell carcinoma is by far the 
most frequent histological form. In our context, despite the early warning signs, a large number of laryngeal cancers are diagnosed at a relatively advanced stage (stage T3 T4). This reduces treatment options, increases the complexity of management, and worsens the prognosis.

\section{Type of study}

This is a single - institutional retrospective Analytical and Descriptive Study of two cohorts of patients diagnosed with locally advanced non-metastatic squamous cell carcinoma of the Larynx classified as T3, T4 associated or not with lymph node involvement and whose management started between January 2014 and December 2018. The first cohort includes patients who have been operated on at the ENT department of Mohamed VI University Hospital of Marrakech and the second cohort includes those who received organ sparing approach by radiotherapy associated or not with chemotherapy: these patients were followed up at the Department of Oncology and Radiotherapy of the same university Hospital.

\section{Inclusion and exclusion criteria}

\section{Group 1: Surgery}

Inclusion criteria: LvPatients with laryngeal squamous cell carcinoma classified as T3, T4 regardless of lymph node status and who have undergone a surgical total laryngectomy

Exclusion criteria: Patients who declined surgical treatment or nonoperable patients

Group 2: Organ preservative treatment with chemoradiotherapy.

Inclusion criteria: In this group were included all patients with squamous cell carcinoma of the larynx classified as T3, T4 irrespective of lymph node involvement and having received radiotherapy associated or not with chemotherapy

Exclusion criteria: Previously operated patients or Patients who have not undergone any treatment, patients with distant metastasis (M1), patients with incomplete records

\section{Data collection}

Data were collected from medical records of patients admitted to the hospital or followed up in consultation during the survey period at the otorhinolaryngology (ENT) and Oncology Department of the University Hospital of Mohamed VI, using a collection form. The selection of patients was carried out using the hospital register of the two departments. The statistical analysis was done using Excel 2013 for data collection and SPSS version 20.0 for data description and analysis. Quantitative data were described by their frequencies and percentages. Quantitative data were described by their means and standard deviation. Survival data were described using the Kaplan and Meier method. The comparisons of the two groups on the epidemiological and clinical characteristics of the patients and the post-treatment follow-up were carried out for the qualitative parameters using a Chi-square or Fisher exact test (when the conditions of validity of the Chi-square test were not verified) and for the quantitative parameters using the Student's T-test. Survival curves were established according to the Kaplan Meier model and the differences observed between the curves were analyzed with the logrank test. The significance level is set at $\mathrm{p}<0.05$.

\section{Consent form}

The surgery or radiochemotherapy was performed after the informed consent of the patients during the pre-anesthesia visit or the radiotherapy referral meeting (Table 1 ).

Table I The comparisons of the two groups on the epidemiological and clinical characteristics of the patients

\begin{tabular}{|c|c|c|c|}
\hline Variable & Surgery & Chemoradiotherapy & $\mathbf{p}$ \\
\hline Age (mean range) & 62 [47 min; $78 \max ]$ & 6I[33 min; $82 \max ]$ & 0.49 \\
\hline Male & $n=61 \quad(96,8 \%)$ & $n=4 I(95,3 \%)$ & 0,69 \\
\hline Female & $n=2(3,2 \%)$ & $n=2(4,7 \%)$ & \\
\hline \multirow[t]{3}{*}{ Tumor location } & Glotto-sus glottic $n=33$ & Glotto-sus glottic $n=24$ & \\
\hline & Glotto-sub glottic $n=6$ & Glotto-sub glottic $n=6$ & 0,62 \\
\hline & All the free floors $n=24$ & All the free floors $n=13$ & \\
\hline \multirow[t]{2}{*}{ Degree of differentiation } & Moderate differentiated $\mathrm{n}=54$ & Moderate differentiated $n=31$ & 0,27 \\
\hline & Well differentiated $n=6$ & Well differentiated $n=6$ & \\
\hline Stage T T3 & $n=33$ & $n=16$ & 0.12 \\
\hline $\mathrm{T} 4$ & $\mathrm{n}=30$ & $\mathrm{n}=27$ & \\
\hline \multirow[t]{6}{*}{ Stage $N$} & No: $n=44$ & No: $n=20$ & \\
\hline & $\mathrm{NI}: \mathrm{n}=8$ & $\mathrm{NI}: \mathrm{n}=10$ & 0.06 \\
\hline & $\mathrm{N} 2 \mathrm{a}: \mathrm{n}=\mathrm{I}$ & $\mathrm{N} 2 \mathrm{a}: \mathrm{n}=3$ & \\
\hline & $N 2 b: n=3$ & $N 2 b: n=6$ & \\
\hline & $\mathrm{N} 2 \mathrm{c}: \mathrm{n}=7$ & $\mathrm{~N} 2 \mathrm{c}: \mathrm{N}=3$ & \\
\hline & N3 $: n=0$ & N3 $: n=1$ & \\
\hline \multirow[t]{3}{*}{ Classification AJCC } & Stage III: $n=29(46 \%)$ & Stage III: $n=9(20,9 \%)$ & 0.008 \\
\hline & Stage IV a: $\mathrm{n}=34$ (54\%) & Stage Iva: $\mathrm{n}=32(74,4 \%)$ & \\
\hline & Stage IV b: $n=0(0 \%)$ & Stage IV b: $n=2(4,7 \%)$ & \\
\hline
\end{tabular}

Citation: Désiré N, Leilla B, Merriam EL, et al. Outcome comparison between radiochemotherapy and surgery in the management of locally advanced nonmetastatic laryngeal cancer a single institution experience.J Otolaryngol ENT Res. 202 I; I3(2):34-4I. DOI: I0.15406/joentr.202I.I3.00487 


\section{Surgical procedure}

The surgery was performed after the informed consent of the patients and a preoperative check-up during the pre-anesthesia consultation. The laryngectomy with the courage of the jugulocarotid lymph nodes was performed bilaterally in all patients either functionally or radically depending on the size of the node.

\section{Radiotherapy}

There were three cases in the radio-chemotherapy group: 4 patients received exclusive radiotherapy, 6 patients received concomitant radio-chemotherapy from the start and 33 patients underwent induction chemotherapy followed by radio-chemotherapy. Intensitymodulated conformal radiotherapy (IMRT) is the reference technique for the treatment of laryngeal cancer. It is recommended because it allows optimal coverage of target volumes and a reduction in the dose delivered to healthy tissues (Organ at risk). The management protocol was as follows: if radio-chemotherapy is indicated (in post-op or very locally advanced tumors that cannot go on surgery operated or if the patient refuses total laryngectomy), Preparation of the treatment: Oral and dental check-up, cessation of alcohol and tobacco intoxication, preventive tracheotomy if the tumor is large.

Acquisition of anatomical data by a dosimetric scanner in the treatment position with a five-point mask and anatomic data was sent to the treatment planning system for target volumes and OAR delineation GTV (gross tumor volume), CTV (clinical tumor volume) PTV (provisional target volume) corresponding to the natural history of laryngeal cancer and tumor volume) and dosimetric validation before the beginning of treatment cessions.

\section{Results}

106 patients were included in the study, 63 patients in the surgery group and 43 patients in the radio-chemotherapy group. The age of our patients ranged from 33 to 82 years, with a mean of 61 years (standard deviation 9.80). There was a predominance of patients in the 51-60 age groups. The majority of patients were male in both treatment groups with $96.23 \%$ of patients male. $88.7 \%$ were smokers with an average consumption of 26.4PA. Alcohol smoking was found in $19 \%$ of cases. The first symptom reported by patients in both groups was dysphonia. At the time of diagnosis, the symptomatology was dominated by dyspnea in $63 \%$. The time between the onset of symptoms and the consultation was on average 14.46 months with extremes ranging from 1 month to 8 years. There was a peak in frequency at 1 year. In our report, all patients underwent direct laryngoscopy with a biopsy of the tumor under general anesthesia. A cervical CT scan with contrast injection to better defines the extension to areas that cannot be easily explored by endoscopy, to establish the tumor's classification, and also to evaluate the lymph node involvement. Extension assessment by imaging and by pan endoscopy to look for other locations in the upper aerodigestive tract and lung metastasis.

The carcinological characteristics of the tumor were defined by clinical examination, endoscopy, and cervicothoracic CT scan, in both groups, the glotto-sus glottic location was in the majority Stage IVA was the predominant in both arms. The two groups were then compared statistically according to local tumor control, local recurrence, lymph node recurrence, and distant metastasis. Early deaths and patients who were lost to follow-up were excluded from the analysis. Tumor control was statistically significant in the surgery group Table 2 .

Table 2 Outcome comparison among those two groups

\begin{tabular}{|c|c|c|c|}
\hline \multirow{2}{*}{ Variables } & Surgery & Chemoradiotherapy & \multirow{2}{*}{$\mathbf{p}$} \\
\hline & n $\%$ & n $\%$ & \\
\hline Local control & $n=47(83,9 \%)$ & $n=12(41,4 \%)$ & $<0,0001$ \\
\hline Local recurence & $n=8(14,5 \%)$ & $n=6(46,2 \%)$ & 0,02 \\
\hline Lymph node recurrence & $\mathrm{n}=2(3,6 \%)$ & $\mathrm{n}=2(15,4 \%)$ & 0,16 \\
\hline Distant Metastasis & $n=7(12,5 \%)$ & $n=5(27,8)$ & 0,13 \\
\hline
\end{tabular}

Table 3 Outcome by stage

\begin{tabular}{llll}
\hline Variables & Stage III & Stage IV & P \\
\hline Local Control & $\mathrm{n}=28(87,5 \%) / 32$ & $\mathrm{n}=3 \mid(58,5 \%) / 53$ & 0,005 \\
Local recurence & $\mathrm{n}=3(10,5 \%) / 29$ & $\mathrm{n}=\mathrm{II}(28,2 \%) / 39$ & 0,07 \\
Lymph node recurence & $\mathrm{n}=\mathrm{I}(3,4 \%) / 29$ & $\mathrm{n}=3(7,7 \%) / 39$ & 0,42 \\
Distant Metastasis & $\mathrm{n}=7(\mid 2,5 \%)$ & $\mathrm{n}=8(\mid 8,2 \%) / 44$ & 0,43 \\
\hline
\end{tabular}

It should be noted that there was a large number of missing data (30 patients) in the radio-chemotherapy group due to a large number of lost to follow-up and early death (Table 2). The comparison between stage III and IV without considering treatment option, we found a better local control with stage III: $87 \%$ versus $58 \%$ for stage IV with a statistically significant difference $(\mathrm{p}=0,005)$. At 1 year, $88 \%$ of patients were alive after surgery compared to $60 \%$ after radiochemotherapy (Table 2). At 3 years overall survival was $77 \%$ for the surgery arm versus $48 \%$ for the radiotherapy $(p=0.005)$ within 5 and 10 missing data respectively for each cohort. The proportion of patients alive without local or lymph node recurrence and metastatic progression at 1 year was $94 \%$ for surgery ( $\mathrm{n}=52$ out of 55 ) versus $84 \%$ ( $n=11$ out of 13 ) for radio-chemotherapy arm, Survival at 3 years was $85 \%$ versus $54 \%$ respectively $(\mathrm{p}=0.05$ ). 


\section{Overall survival}

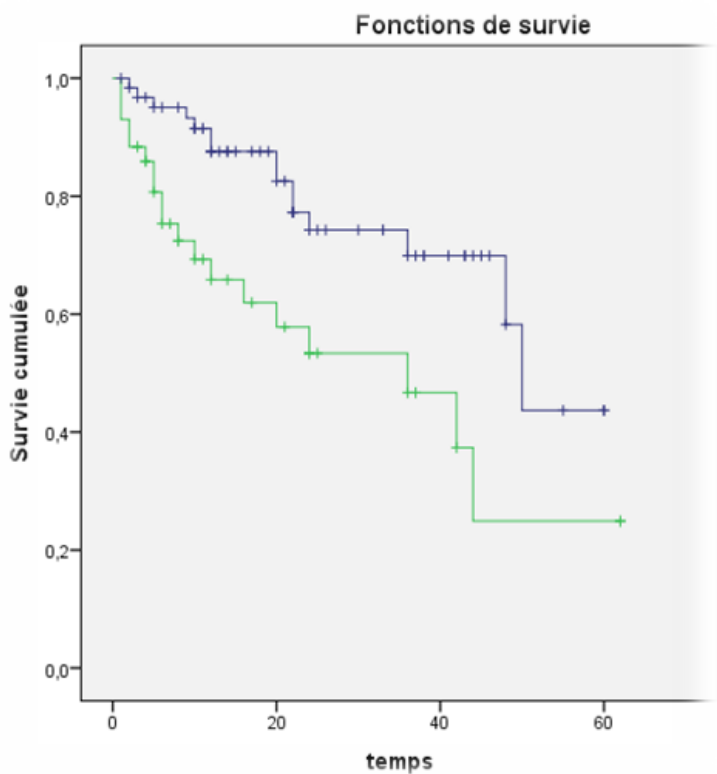

Recurrence-free survival

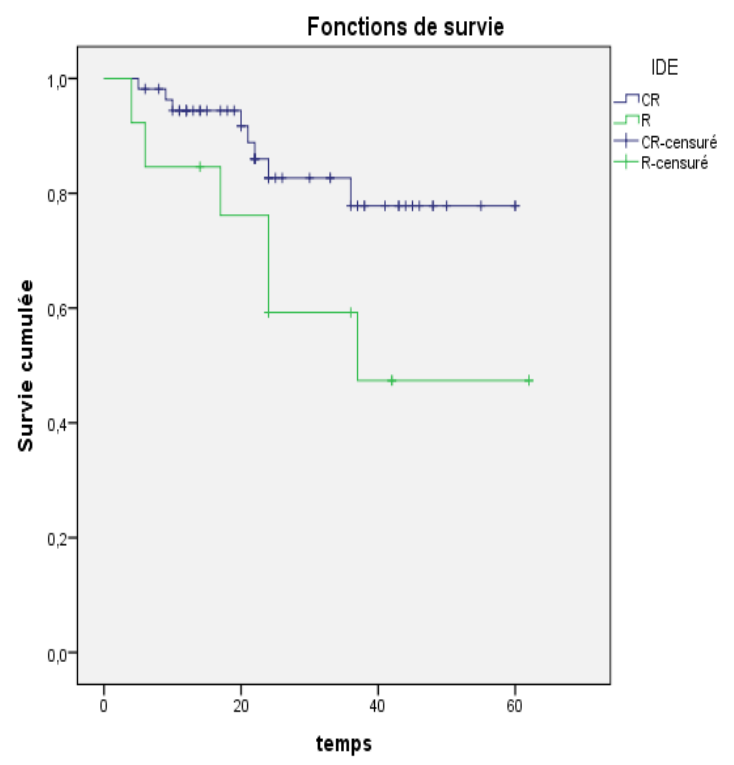

\section{Discussion}

\section{Epidemiology}

Incidence and mortality: Laryngeal cancer ranks 14th in men and 26th in women. In Morocco, according to the registry of cancer the Grand Casablanca, this cancer represents $2 \%$ of all cancer sites recorded between 2008 and 2012. Mortality related to laryngeal cancer worldwide is estimated in 2018 at 94771 deaths per year with still a clear male predominance. ${ }^{1}$

Age-sex: Laryngeal cancers occur between the ages of 45 and 70 , rare before the age of 40, and exceptional in children (they are mostly embryonic tumors).

The average age varies according to the authors comparing the different treatment methods. In our study, the average age was 61 years with a clear male predominance. This is in agreement with the different series in the literature. ${ }^{2}$

\section{Risk factors}

There are several exogenous factors implicated in laryngeal cancer but tobacco and the alcohol-smoking combination remains the most significant factor. Several studies have documented the role of tobacco in the initiation of laryngeal squamous cell carcinoma. ${ }^{3,4}$ Its carcinogenic effects are linked to different factors: ${ }^{2}$ Its irritative eeffect linked to some components of the smoke (phenols, aldehydes, effects on the vascular network, and the dependence of subjects (nicotine), unquestionably carcinogenic agents: polycyclic aromatic hydrocarbons and nitrosamines. As for alcohol, its role remains difficult to specify. The main compound, ethanol, is not carcinogenic in itself. It could act at the level of liver microsomes and compromise the metabolism of genuine carcinogens such as those in tobacco. ${ }^{2}$ Other risk factors (occupational, genetic, nutritional, viral) not studied in our report have been incriminated by other studies. ${ }^{3,5,6}$ In our study, the majority of patients had a smoking addiction $(85.71 \%)$, whether or not they had weaned themselves off tobacco. Alcohol-smoking synergy was observed in $19 \%$ of cases.

\section{Results of our study compared with the literature}

There is some controversy regarding the optimal treatment approach for patients with advanced laryngeal cancer. Combinations of radiotherapy and chemotherapy as alternatives to total laryngectomy have been promoted to encourage the preservation of laryngeal function in these patients. However, the results of randomized clinical trials of surgical and non-surgical approaches have failed to show a significant improvement in overall survival and recurrencefree survival or a clear superiority of one therapeutic approach over another. $^{7}$

Despite this, concomitant chemo-radiotherapy approaches have been widely adopted due to the desire for preservation of this organ, and evidence from meta-analyses showed that concomitant chemo-radiotherapy is superior to radiotherapy alone and may also be superior to neo-adjuvant or sequential chemotherapy combined with radiotherapy, ${ }^{8,9}$ both surgical and non-surgical approaches have resulted in 5-year survival rates of less than $50 \%$. Although laryngeal preservation rates with concurrent radio-chemotherapy are generally higher than those achieved with a sequential approach. ${ }^{10}$

No organ-sparing approach has achieved higher survival rates than total laryngectomy. There is also concern that long-term toxicity, cost, and treatment-related deaths are greater than with total laryngectomy. ${ }^{11,12}$ In addition, the survival of patients enrolled in carefully controlled chemotherapy clinical trials may not apply to an unselected patient population or replicated outside the clinical trial setting. ${ }^{13,14}$ Our study aimed to compare the carcinological results as well as the overall survival and recurrence-free survival at 1 and 3 years in the two groups. The first one concerned patients who received surgical treatment and the second one radio-chemotherapy protocols. We first investigated whether the two groups were comparable for several epidemiological and clinical characteristics. We found no statistically significant difference in terms of age, gender, tumor location, degree of differentiation, T-stage, and N-stage. The only statistically significant difference was in the AJCC stage with an increase in stage IVa in the radio-chemotherapy group (74.4\%) compared to surgery (54\%). This may partly explain the better overall survival and recurrence-free survival results in the surgery group compared to radio-chemotherapy. 


\section{Local control}

The results of clinical studies focus on local control as a predictor of a good response to a cancer treatment strategy. In the literature, a better rate of local control with the surgical strategy than with the nonsurgical strategy has been observed in most studies. In our study, more than half of the patients treated with surgery achieved locoregional tumor control in $83.9 \%$ compared to $41.4 \%$ for radio-chemotherapy strategies $(\mathrm{p}<0.001)$. When analyzing the local control between stage III and IV, without considering treatment, it was found to be better with stage III. It can be concluded that the advanced stage is an unfavorable prognostic factor for local control. These results are in line with those of Vengalil et al. ${ }^{15}$ who evaluated the clinical outcome in patients with T4 laryngeal cancer treated with primary radiotherapy versus primary laryngectomy. There was a trend towards better local control with surgery $88 \%$ versus $74 \%$ for radio-chemotherapy $(\mathrm{p}=0.02)$. Smoking and cartilage damage were unfavorable prognostic factors for local control in multivariate analysis.

For Rosenthal et al. ${ }^{16}$, initial local tumor control was $83 \%$ for surgery versus $63 \%$ for radio-chemotherapy $(p<0.007)$, but after salvage surgery, for $38 \%$ of patients with local recurrence the two groups were comparable in terms of local tumor control with a $\mathrm{p}=0.5$. Others obtained the same results but the difference was not statistically significant. Rades et al. ${ }^{17}(75 \%$ versus $66 \% \mathrm{p}=0.39)$, Hsin et al. ${ }^{18}(80 \%$ versus $45.2 \% \mathrm{P}=0.105)$. Both studies found tumor site and tumors volume greater than $15 \mathrm{~cm}^{3}$ respectively as factors influencing local control. Nguyen-Tan et al. ${ }^{19}$ conducted a retrospective study reviewing the records of 223 patients treated for T3-T4 squamous cell carcinoma of the larynx between 1 October 1957 and 1 December 1999. They found that the tumor control rate was better with surgery with $72 \%$ locoregional control compared to $43 \%$ over 5 years for radio-chemotherapy. For their part, advanced nodal involvement and low hemoglobin level were a poor prognosis for locoregional control.

\section{Local and lymph node recurrence}

One of the major issues in monitoring patients treated for VADS cancer is the occurrence of local or lymph node recurrence. Recurrence is defined as the reappearance of the tumor after a period of complete remission. Early diagnosis of recurrences that occur less than 6 months after treatment should allow curative treatment to be proposed and be accompanied by an improvement in survival. ${ }^{20}$ Twenty-five percent of recurrences occur within the first 3 years after initial curative treatment, $2 / 3$ of which are local. ${ }^{21}$ Recurrence of laryngeal cancer is successfully treated in about $85 \%$ of cases..$^{22}$ The prognosis is correlated with the $\mathrm{T}$ and $\mathrm{N}$ stages and in particular with the lymph node status. ${ }^{23}$ In our series, $46.2 \%$ of patients in the radio-chemotherapy arm developed local tumor recurrence compared to only $14.5 \%$ for surgery $p=0,02$. The rate of recurrence between stages III and IV was statistically insignificant. It should be noted that the high number of loss to follow-up and death during treatment in the radio-chemotherapy group was a negative factor on the accuracy of the presence or absence of recurrence.

In the literature, there are not many studies that address local and lymph node recurrence. In the veterans' trial, ${ }^{24}$ local recurrence was significantly more frequent in the radio-chemotherapy group with $12 \%$ compared to $2 \%$ in the surgery group $p=0.001$. the recurrence rate in the surgery arm remains high in our study (14.5\%) compared to the literature. This can be explained by the median time between surgery and radiotherapy which is 5 months in our series. This delay is very high, especially as it has been shown that starting radiotherapy more than 6 weeks after surgery increases the risk of local recurrence by $2.29 .{ }^{25}$ This study included all head and neck cancers and did not differentiate between laryngeal and other locations. A radiobiology study showed that a delay of 4 weeks before starting radiotherapy increases the number of cancer cells by $67 \%$ and decreases the chances of local control by $8 \%{ }^{26}$ The French INCa guidelines for head and neck tumors are based on this type of study to establish the acceptable delay between surgery and radiotherapy, which should be between 4 and 6 weeks. ${ }^{27}$ This is in line with those in Canada, the UK, or Denmark. ${ }^{28,29}$ Since local control and recurrence are closely related, it can be considered that in all studies where local control was superior in the surgery arm, there was more local recurrence in the radio-chemotherapy arm, although the difference may not be statistically significant.

\section{Distant metastasis}

The preferred metastasis site is the lung, followed by bone and liver. In more than three-quarters of cases, the pulmonary metastatic site is unique. Other metastatic sites are associated with lung involvement in $97 \%$ of cases. ${ }^{30}$ Metastatic progression occurs during the first two years of follow-up with a median of 10 months. It is rare to find metastases after 3 years of follow-up and a second primary should be considered in case of late metastases. Factors favoring the occurrence of metastases are lymph node status $(>\mathrm{N} 2 \mathrm{~b})$, the presence of capsular rupture, vascular, lymphatic, or perineural emboli. ${ }^{30}$

Regarding the rate of metastasis according to treatment, in our series no statistically significant difference was found between the two treatment strategies with $13.8 \%$ of metastatic progression for surgery and $18.2 \%$ for radio-chemotherapy $p=0.43$. In the veterans' study, ${ }^{24}$ recurrence rates in the cervical lymph nodes were similar in both groups, but distant metastases occurred in $17 \%$ of patients in the surgery group compared to $2 \%$ in the chemotherapy group $(\mathrm{p}=0.001)$.

\section{Overall survival}

Our study shows that patients with advanced laryngeal cancer treated with surgical therapy have better survival than patients treated with non-surgical therapy. Since the advent of non-surgical therapies, several studies have found a decrease in overall survival in laryngeal cancer. ${ }^{31,32}$ Cosetti et al. ${ }^{31}$ examined survival trends in laryngeal cancer from 1977 to 2002, using the SEER database. They found that relative survival worsened over time for patients with distant supraglottic cancer and patients with glottic cancer. Dziegielewski et al..$^{33}$ reviewed 258 cases of T3 and T4a laryngeal cancer diagnosed between 1998 and 2008 using the Alberta Cancer Registry.

As in our study, they found that patients treated with total laryngectomy plus radiotherapy and/or chemotherapy had longer overall survival than patients treated with chemotherapy or radiotherapy alone. Similarly, O'Neil et al ${ }^{34}$ studied treatment-related complications and survival in elderly patients with stage III or IV laryngeal cancer using data from the SEER cancer registry combined with health insurance claims. Similar to our study, they found that total laryngectomy resulted in an $18 \%$ lower risk of death after adjustment for propensity to receive treatment. Megwalu et al. ${ }^{35}$ evaluated the survival outcomes of laryngeal conservation versus surgical therapy for advanced laryngeal cancer in the United States using a large cancer database based on a population of 5394 patients diagnosed with stage III or IV laryngeal squamous cell carcinoma1992 2009. They found that patients with advanced laryngeal cancers treated with surgical therapy had better survival than patients treated with nonsurgical therapy. 
An EORTC Group phase III head and neck trial ${ }^{36}$ involving 194 eligible patients with piriform sinus cancer compared surgery with postoperative radiotherapy to patients who responded to induction chemotherapy with cisplatin/5-fluorouracil and received $70 \mathrm{~Gy}$ of radiotherapy after three courses of chemotherapy. The two treatments were found to be equivalent in terms of survival. Other studies have found equivalent survival between the two types of treatment over time but remains significant., $77,37,38$

\section{Tumor-free survival}

In most studies comparing surgery and radio-chemotherapy, overall survival and tumor-free survival results are similar. In our study, there was no statistically significant difference between the groups in terms of tumor-free survival, with 3-year survival rates of $85.4 \%$ for surgery and $53 . \%$ for chemoradiotherapy $p=0.05$. It should be noted that there was a large number of missing data in the radiochemotherapy group, mainly due to a large number of deaths and loss of sight in the first year, with no indication of the presence or absence of recurrence, compared to 8 missing data for surgery. The same results have been observed in most studies in the literature. Bryant et al. ${ }^{39}$ found $33 \%$ for radiotherapy followed by salvage surgery in case of failure and $54 \%$ for surgery followed or not by radiotherapy at 5 years with no statistically significant difference $p=2.93$.

Rades et al. ${ }^{17}$ reported metastasis-free survival rates of $76 \%$ and $77 \%(\mathrm{p}=0.76)$ at 5 years for surgery and radiochemotherapy respectively. In a univariate analysis, gender $(\mathrm{p}=0.035)$, tumor site $(\mathrm{p}=0.003)$, histological grade $(\mathrm{p}<0.001)$ and $\mathrm{N}$ category $(\mathrm{p}=0.021)$ were associated with metastasis-free survival. For Hsin et $\mathrm{al}^{38}$ the progression-free survival rate was higher in the surgery group than in the radio-chemotherapy group with respectively $76.9 \%$ versus $46.1 \%$ at 5 years, however, the difference was not statistically significant $p=$ 0.12 . Rosenthal et al. ${ }^{16}$ reported a 5 -year recurrence-free survival rate of $60 \%$ for surgery and $48.5 \%$ for chemoradiotherapy with $p=0.1$. In our retrospective study of 106 patients, no significant difference in overall survival was observed between T3 and T4 laryngeal cancers, or between stage III and IV disease. Historically, survival of T3 laryngeal cancers has been better than T4 laryngeal cancers. Robin et al. ${ }^{40}$ found that of all patients treated with total laryngectomy, patients classified as $\mathrm{T} 3 \mathrm{had}$ better survival than those classified as T4 (supraglottis T3N0: 83\%; total 22 cases; T4N0: $45 \%$; total 10 cases; glottis: T3N0: 50\%; total 107 cases; and T4N0: 39\%; total 9 cases). Groome et al. ${ }^{41}$, comparing different TNM-based stage groups in laryngeal cancer using data from Canada and Norway, reported a mortality rate for laryngeal cancer of 5.4 and $7.5 \%$ for T3N0, and 10.5 and $9.0 \%$ for T4N0 (for Canada and Norway, respectively).

However, the authors did not report treatment, but including all T, $\mathrm{N}$, and $\mathrm{M}$ classifications, more than $80 \%$ of patients in both groups were irradiated. It is therefore interesting to note that this difference in survival was absent in our cohort. Regarding the treatment protocol between the two tumor stages, a meta-analysis by Zhao-Xian Tang et al ${ }^{42}$ of 15 studies compared the curative effects between total laryngectomy and radiochemotherapy. They found that between the two treatment strategies there was no significant difference in survival for patients classified as T3. The superiority of surgery over radiochemotherapy was significant for T4 patients. They concluded that total laryngectomy should be used for $\mathrm{T} 4$ patients and laryngeal preservation protocols for $\mathrm{T} 3$ patients. The same conclusions were drawn by another meta-analysis of 16 studies by Xiaoyuan $\mathrm{Fu}$ et $\mathrm{al}^{43}$. More recent studies ${ }^{44,45}$ came to the same conclusion: they found that patients with T4 disease had significantly better survival after total laryngectomy $(55 \%)$ than after radiochemotherapy $(25 \%)$ or radiotherapy alone $(0 \%)$. Furthermore, after controlling for lymph node status, organ-sparing treatment remained a significant predictor of poorer survival. ${ }^{46-55}$

\section{The limits of our study}

Due to its retrospective nature, we do not have complete information for the patients. The sample sizes between the two groups were not equivalent in the two groups, which is mainly due to a large number of treatment discontinuations before the end of the protocol in the radio-chemotherapy group and the heterogeneity of treatment received in the radio-chemotherapy group.

\section{Conclusion}

The optimal treatment for advanced squamous cell carcinoma of the larynx is highly controversial. Total laryngectomy remains the gold standard of treatment but organ-sparing protocols have proven to be as effective as surgical therapy. However, in our study, total laryngectomy plus lymph node dissection showed better efficacy in terms of locoregional control and significantly increased overall survival and recurrence-free survival. This makes surgery the treatment of choice for T3 and T4 in our single-institution setting. Possible reasons for these results may be poor patient selection, inadequate follow-up, incomplete treatment, and interrupted treatment sessions but also the long delay in consultation. Patients should be made aware of the small but significant disadvantage of non-surgical therapy as part of the shared decision-making process when selecting treatment. Both surgery and radio-chemotherapy can be effective if the treatment indications are well directed. ${ }^{56-65}$ These indications depend on a number of parameters and should be considered at the multidisciplinary consultation meetings (RCP) and adapted on a case-by-case basis. Further large randomized prospective studies are needed to confirm or affirm our results.

\section{Acknowledgments}

None.

\section{Funding}

None.

\section{Conflicts of interest}

None.

\section{References}

1. Cancers du Larynx. Elsevier. 2019.

2. Lefebvre JL, Chevalier D. Cancers du larynx. EMC - Oto-rhinolaryngologie. 2005;2(4):432-457.

3. Gallus S, Bosetti C, Franceschi S, et al. Laryngeal cancer in women: tobacco, alcohol, nutritional, and hormonal factors. Cancer Epidemiol Biomarkers Prev. 2003;12(6):514-517.

4. Morales SVMM, Llopis GA, Moreno GE, et al. Toxic habits concerning cancer of the larynx. Acta Otorrinolaringol Esp. 1997;48(1):45-50.

5. Shan'gina OV, Sdvizhkov AM, Finkel'shtern MR, et al. Risk factors of laryngeal cancer in Central and Eastern Europe. Vopr Onkol. 2007;53(3):321-328.

6. Righini CA, Karkas A, Morel N, et al. Risk factors for cancers of the oral cavity, pharynx (cavity excluded), and larynx. Presse Med. 2008;37(9):1229-1240. 
7. Wolf GT, Bellile E, Eisbruch A, et al. Survival Rates Using Individualized Bioselection Treatment Methods in Patients With Advanced Laryngeal Cancer. JAMA Otolaryngol Head Neck Surg. 2017;143(4):355-366.

8. Pignon JP, Bourhis J, Domenge C, et al. Chemotherapy added to locoregional treatment for head and neck squamous cell carcinoma: three meta-analyses of updated individual data. MACH-NC Collaborative Group. Meta-Analysis of Chemotherapy on Head and Neck Cancer Lancet. 2000;355(9208):949-955.

9. Haddad R, O’NeillA, Rabinowitz G, etal. Induction chemotherapy followed by concurrent chemoradiotherapy (sequential chemoradiotherapy) versus concurrent chemoradiotherapy alone in locally advanced head and neck cancer (PARADIGM): a randomized phase 3 trial. Lancet Oncol. 2013;14(3):257-264.

10. Forastiere AA, Goepfert H, Maor M, et al. Concurrent Chemotherapy and Radiotherapy for Organ Preservation in Advanced Laryngeal Cancer. New England Journal of Medicine. 2003;349(22):2091-2098.

11. Strojan P, Haigentz M, Bradford CR, et al. Chemoradiotherapy vs. total laryngectomy for primary treatment of advanced laryngeal squamous cell carcinoma. Oral Oncol. 2013;49(4):283-286.

12. Haigentz M, Vermorken JB, Forastiere AA, et al. When is chemotherapy in head and neck squamous cell carcinoma not indicated? Eur Arch Otorhinolaryngol. 2015;272(4):781-787.

13. Sanabria A, Chaves ALF, Kowalski LP, et al. Organ preservation with chemoradiation in advanced laryngeal cancer: The problem of generalizing results from randomized controlled trials. Auris Nasus Larynx. 2017;44(1):18-25.

14. Chavez-MacGregor M, Giordano SH. Randomized Clinical Trials and Observational Studies: Is There a Battle? J Clin Oncol. 2016;34(8):7727723

15. Vengalil S, Giuliani ME, Huang SH, et al. Clinical outcomes in patients with T4 laryngeal cancer treated with primary radiotherapy versus primary laryngectomy. Head Neck. 2016;38 Suppl 1:E2035-E2040.

16. Rosenthal DI, Mohamed ASR, Weber RS, et al. Long-term outcomes after surgical or nonsurgical initial therapy for patients with T4 squamous cell carcinoma of the larynx: A 3-decade survey. Cancer. 2015;121(10):16081619

17. Rades D, Schroeder U, Bajrovic A, et al. Radiochemotherapy versus surgery plus radio(chemo)therapy for stage T3/T4 larynx and hypopharynx cancer - results of a matched-pair analysis. Eur J Cancer. 2011;47(18):2729-2734.

18. Hsin LJ, Fang TJ, Tsang NM, et al. Tumor volumetry as a prognostic factor in the management of T4a laryngeal cancer. Laryngoscope. 2014;124(5):1134-1140.

19. Nguyen-Tan PF, Le QT, Quivey JM, et al. Treatment results and prognostic factors of advanced T3-4 laryngeal carcinomas: the University of California, San Francisco (UCSF) and Stanford University Hospital (SUH) experience. Int J Radiat Oncol Biol Phys. 2001;50(5):1172-1180.

20. Ritoe SC, Krabbe PFM, Kaanders JHAM, et al. Value of routine followup for patients cured of laryngeal carcinoma. Cancer. 2004;101(6):13821389

21. Boysen M, Lövdal O, Tausjö J, et al. The value of follow-up in patients treated for squamous cell carcinoma of the head and neck. Eur J Cancer. 1992;28(2-3):426-430.

22. Davidson J, Keane T, Brown D, et al. Surgical salvage after radiotherapy for advanced laryngopharyngeal carcinoma. Arch Otolaryngol Head Neck Surg. 1997;123(4):420-424.

23. Goodwin WJ. Salvage surgery for patients with recurrent squamous cell carcinoma of the upper aerodigestive tract: when do the ends justify the means? Laryngoscope. 2000;110(3 Pt 2 Suppl 93):1-18.
24. Department of Veterans Affairs Laryngeal Cancer Study Group, Wolf GT, Fisher SG, et al. Induction chemotherapy plus radiation compared with surgery plus radiation in patients with advanced laryngeal cancer. $N$ Engl J Med. 1991;324(24):1685-1690.

25. Huang J, Barbera L, Brouwers M, et al. Does delay in starting treatmen affect the outcomes of radiotherapy? A systematic review. J Clin Oncol. 2003;21(3):555-563.

26. Sharp L, Lewin F, Hellborg H, et al. When does my treatment start?The continuum of care for patients with head and neck cancer. Radiother Oncol. 2002;63(3):293-297.

27. Institut National Du Cancer.

28. Bradley PJ, Zutshi B, Nutting CM. An audit of clinical resources available for the care of head and neck cancer patients in England. J Laryngol Otol. 2005;119(8):620-626.

29. .Groome PA, O’Sullivan B, Mackillop WJ, et al. Laryngeal cancer treatment and survival differences across regional cancer centers in Ontario, Canada. Clin Oncol (R Coll Radiol). 2011;23(1):19-28.

30. De Bree R, Deurloo EE, Snow GB, et al. Screening for distant metastase in patients with head and neck cancer. Laryngoscope. 2000;110(3 Pt 1):397-401.

31. .Cosetti M, Yu G-P, Schantz SP. Five-Year Survival Rates and Time Trends of Laryngeal Cancer in the US Population. Arch Otolaryngol Head Neck Surg. 2008;134(4):370-379.

32. Hoffman HT, Porter K, Karnell LH, et al. Laryngeal cancer in the United States: changes in demographics, patterns of care, and survival. Laryngoscope. 2006;116(9 Pt 2 Suppl 111):1-13.

33. Dziegielewski PT, O'Connell DA, Klein M, et al. Primary total laryngectomy versus organ preservation for T3/T4a laryngeal cancer: a population-based analysis of survival. J Otolaryngol Head Neck Surg. 2012;41 Suppl 1:S56-S64.

34. O’Neill CB, O’Neill JP, Atoria CL, et al. Treatment complications and survival in advanced laryngeal cancer: a population-based analysis. Laryngoscope. 2014;124(12):2707-2713.

35. Megwalu UC, Sikora AG. Survival outcomes in advanced laryngeal cancer. JAMA Otolaryngol Head Neck Surg. 2014;140(9):855-860.

36. Forastiere AA, Zhang Q, Weber RS, et al. Long-term results of RTOG 91-11: a comparison of three nonsurgical treatment strategies to preserve the larynx in patients with locally advanced larynx cancer. J Clin Oncol. 2013;31(7):845-852.

37. Timmermans AJ, de Gooijer CJ, Hamming-Vrieze O, et al. T3-T4 laryngeal cancer in The Netherlands Cancer Institute; 10-year results of the consistent application of an organ-preserving/-sacrificing protocol. Head Neck. 2015;37(10):1495-1503.

38. Timme DW, Jonnalagadda S, Patel R, et al. Treatment Selection for T3/ T4a Laryngeal Cancer: Chemoradiation Versus Primary Surgery. Ann Otol Rhinol Laryngol. 2015;124(11):845-851.

39. Bryant GP, Poulsen MG, Tripcony L, et al. Treatment decisions in T3N0M0 glottic carcinoma. Int J Radiat Oncol Biol Phys. 1995;31(2):285-293.

40. Robin PE, Rockley T, Powell DJ, et al. Survival of cancer of the larynx related to treatment. Clin Otolaryngol Allied Sci. 1991;16(2):193-197.

41. Groome PA, Schulze K, Boysen M, et al. A comparison of published head and neck stage groupings in laryngeal cancer using data from two countries. J Clin Epidemiol. 2002;55(6):533-544.

42. Tang ZX, Gong JL, Wang YH, et al. Efficacy comparison between primary total laryngectomy and nonsurgical organ- preservation strategies in the treatment of advanced-stage laryngeal cancer: A meta-analysis. Medicine (Baltimore). 2018;97(21):e10625.60. 
43. Xiaoyuan FU, Qi Zhou D, Xianquan Z. Efficacy Comparison Between Total Laryngectomy and Nonsurgical Organ- Preservation Modalities in Treatment of Advanced Stage Laryngeal Cancer: A Meta-Analysis. 2016;95(14):e3142.

44. Bates JE, Amdur RJ, Morris CM, et al. Curative-dose Chemoradiotherapy Versus Total Laryngectomy For Stage T3-T4 Squamous Cell Carcinoma of the Larynx: An "Apples-to-Apples" Analysis of the National Cancer Database. Am J Clin Oncol. 2019;42(6):527-533.

45. Gourin CG, Conger BT, Sheils WC, et al. The effect of treatment on survival in patients with advanced laryngeal carcinoma. Laryngoscope. 2009;119(7):1312-1317.

46. Marandas P. Les cancers des VADS dans l'histoire. Annales françaises d'Oto-rhino-laryngologie et de Pathologie Cervico-faciale. 2011;128(2):116-121.

47. Laccourreye H. Traitement chirurgical du cancer du larynx au cours du XX e siècle. 2008.

48. Laccourreye O, Werner A, Maldent JB. Une histoire de la laryngectomie à travers les siècles. 2010.

49. Constable WC, White RL, El-Mahdi AM, et al. Radiotherapeutic management of cancer of the glottis, The University of Virginia, 19561971. Laryngoscope. 1975;85(9):1494-1503.

50. Ogura JH, Sessions DG, Spector GJ. Analysis of surgical therapy for epidermoid carcinoma of the laryngeal glottis. Laryngoscope. 1975;85(9):1522-1530

51. Hawkins NV. The treatment of glottic carcinoma: an analysis of 800 cases. Laryngoscope. 1975;85(9):1485-1493.

52. Vermorken JB, Mesia R, Rivera F, et al. Platinum-based chemotherapy plus cetuximab in head and neck cancer. $N$ Engl J Med. 2008;359(11):11161127.

53. 10.Peyrade F, Cupissol D, Geoffrois L, et al. Systemic treatment and medical management of metastatic squamous cell carcinoma of the head and neck: review of the literature and proposal for management changes. Oral Oncol. 2013;49(6):482-491.

54. Ang KK. Larynx preservation clinical trial design: summary of key recommendations of a consensus panel. Oncologist. 2010;15 Suppl 3:2529
55. Lefebvre J-L, Ang KK, Larynx Preservation Consensus Panel. Larynx preservation clinical trial design: key issues and recommendations-a consensus panel summary. Int J Radiat Oncol Biol Phys. 2009;73(5):12931303.

56. Registre des Cancers de la Région du Grand Casablanca 2008-2012.

57. Bussu F, Miccichè F, Rigante M, et al. Oncologic outcomes in advanced laryngeal squamous cell carcinomas treated with different modalities in a single institution: a retrospective analysis of 65 cases. Head Neck. 2012;34(4):573-579.

58. Grover S, Swisher-McClure S, Mitra N, et al. Total Laryngectomy Versus Larynx Preservation for T4a Larynx Cancer: Patterns of Care and Survival Outcomes. Int J Radiat Oncol Biol Phys. 2015;92(3):594-601.

59. Laccourreye O, Malinvaud D, Ménard M, et al. Total laryngectomy or laryngeal preservation for advanced laryngeal cancer. Impact of the functional risk upon the patient's preferences. European Annals of Otorhinolaryngology, Head and Neck Diseases. 2014;131(2):93-97.

60. Bosetti C, Gallus S, Peto R, et al. Tobacco smoking, smoking cessation, and cumulative risk of upper aerodigestive tract cancers. Am J Epidemiol. 2008;167(4):468-473.

61. Cohen EEW, Karrison TG, Kocherginsky M, et al. Phase III randomized trial of induction chemotherapy in patients with N2 or N3 locally advanced head and neck cancer. J Clin Oncol. 2014;32(25):2735-2743.

62. Primdahl H, Nielsen AL, Larsen S, et al. Changes from 1992 to 2002 in the pretreatment delay for patients with squamous cell carcinoma of larynx or pharynx: a Danish nationwide survey from DAHANCA. Acta Oncol. 2006;45(2):156-161.

63. Chen AY, Fedewa S, Zhu J. Temporal trends in the treatment of early- and advanced-stage laryngeal cancer in the United States, 1985-2007. Arch Otolaryngol Head Neck Surg. 2011;137(10):1017-1024.

64. Brizel DM, Esclamado R. Concurrent chemoradiotherapy for locally advanced, nonmetastatic, squamous carcinoma of the head and neck: consensus, controversy, and conundrum. J Clin Oncol. 2006;24(17):26122617 .

65. Maddox PT, Davies L. Trends in total laryngectomy in the era of organ preservation: a population-based study. Otolaryngol Head Neck Surg. 2012;147(1):85-90 